\title{
Pregnancy and autoimmune connective tissue disorders
}

\author{
Carmeline Motha' ${ }^{1}$, Thilina S Palihawadana ${ }^{2}$, Alastair McKelvey ${ }^{3}$
}

\begin{abstract}
A utoimmune connective tissue disorders include a wide spectrum of diseases, which can have important implications in pregnancy. Firstly, they are common among females of reproductive age, and with few exceptions, are associated with normal fertility. Pregnancy may modify the disease status and the disease may have effects on the pregnant woman and fetus. Normal physiological changes in pregnancy may affect the accuracy and laboratory values of diagnostic tests. Drug therapy for these conditions include immunomudulators, some of which exhibit teratogenicity and are contraindicated in pregnancy and during breastfeeding, while others are safe and can be used if the benefit outweigh the risk. In this review we will specifically discuss, systemic lupus erythematosus (SLE) and secondary antiphospholipid syndrome (APS), rheumatoid arthritis and systemic sclerosis. In all cases, pre-pregnancy counselling is important to ensure that disease control can be optimised before conception. Disease activity should be assessed and drug therapy modified prior to pregnancy. This provides an opportunity for a woman to be counselled about her specific risk of complications in pregnancy. When pregnant, care should be coordinated by a specialist multidisciplinary team including, obstetricians, obstetric physicians, haematologists and rheumatologists.
\end{abstract}

\footnotetext{
${ }^{1}$ Consultant Physician and Senior Lecturer, Faculty of Medicine, University of Kelaniya, Sri Lanka.

${ }^{2}$ Clinical Fellow,

${ }^{3}$ Consultant in Fetal and Maternal Medicine, Norfolk and Norwich University Hospital, Norwich, UK.

Correspondence: Carmeline Motha

E-mail: cmotha6@gmail.com

Competing interests: None
}

\section{Systemic lupus erythematosus}

SLE is an autoimmune disease of unknown aetiology. Many immunological pathways are considered to be involved in the pathophysiology and these are known to include dysfunction of the T cells, B cells, dendritic cells, macrophages and neutrophils, secondary to genetic and/or environmental factors. It is a disease of clinical heterogeneity and currently a formal diagnosis is made in the presence of 4 or more of the 11 criteria described by the American College of Rheumatology in $1982^{1}$ and further updated in 1997 as shown in box $1^{2}$. SLE known to affect multiorgans is known to have phases of active disease and remissions. A widespread hypothesis is that an environmental trigger (such as sunburn or a viraemia) in a genetically susceptible person may initiate the disease process ${ }^{3}$.

SLE is a common autoimmune disease that is encountered in pregnancy. It has a prevalence rate of about 1 per 150 women of childbearing age ${ }^{4}$, and is associated with increased maternal and fetal/neonatal morbidity. There is a racial variation in prevalence, with increased prevalence among Afro-caribbeans followed by Asians.

There remains debate about whether there is an increased incidence of disease exacerbations during pregnancy. A prospective observation of 40 pregnancies in women with SLE Petri et al described a flare up rate in around $60 \%$, although most of these were mild to moderate flares ${ }^{5}$. However, in a case control series, Lockshin et al found no significant difference in flare up rates with a flare up rate of only $13 \%$ being observed when SLE specific signs and symptoms were taken into account ${ }^{6}$. Disease exacerbation may present as constitutional symptoms, renal disease or skin and joint involvement while the risk is associated with the disease state at conception. This ranges from $7-33 \%$ in a woman who has been in disease remission for at least six months prior to conception to $61-67 \%$ if had active disease ${ }^{7,8}$. Presence of renal involvement is also associated with a higher incidence of disease flare up.

The diagnosis of a SLE exacerbation can be difficult in pregnancy due to considerable overlap of normal pregnancy symptoms with features of SLE. Facial erythema, arthralgia, anaemia and a degree of thrombocytopenia may occur in normal pregnancy and can be misinterpreted as disease flare in a woman with lupus. A palpable facial rash, synovitis, haemoglobin count of $<10 \mathrm{~g} / \mathrm{dl}$ not coinciding with the time of maximum haemodilution of pregnancy, and moderate $\left(<100,000 / \mathrm{mm}^{3}\right)$ rather than mild thrombocytopaenia should alert the clinician on the possibility of a lupus flare. SLE is associated with an increase risk of hypertensive disorders of pregnancy. Discriminating preeclampsia form a flare of renal lupus during pregnancy is challenging though vital in the management. Presence of RBC or RBC casts along with proteinuria is more suggestive of a SLE flare up as preeclampsia is known to lead to proteinuria in isolation. Other helpful findings include rising titres of anti-ds DNA and low serum complement values (C3 and C4 levels) as complement levels are often normal in preeclampsia9.

Pregnancy complications of SLE include hypertensive disease of pregnancy, intrauterine growth restriction, preterm delivery, caesarean section, postpartum haemorrhage, neonatal death and neonatal lupus ${ }^{10,11}$. The complication rates are more common in the presence of antiphospholipid antibodies (aPL), of which the presence of lupus anticoagulant (LA) is associated with the highest risk of fetal loss ${ }^{12}$. Factors that confer a higher 


\section{Box 1. 1997 update of the 1982 American College of Rheumatology revised criteria for classification of systemic lupus erythematosus ${ }^{2}$}

\begin{tabular}{|c|c|}
\hline Criterion & Definition \\
\hline 1. Malar rash & Fixed erythema, flat or raised, over the malar eminences, tending to spare the nasolabial folds \\
\hline 2. Discoid rash & $\begin{array}{l}\text { Erythematous raised patches with adherent keratotic scaling and follicular plugging; atrophic } \\
\text { scarring may occur in older lesions }\end{array}$ \\
\hline 3. Photosensitivity & Skin rash as a result of unusual reaction to sunlight, by patient history or physician observation \\
\hline 4. Oral ulcers & Oral or nasopharyngeal ulceration, usually painless, observed by physician \\
\hline 5. Nonerosive arthritis & Involving 2 or more peripheral joints, characterized by tenderness, swelling, or effusion \\
\hline 6. Pleuritis or pericarditis & $\begin{array}{l}\text { Pleuritis - convincing history of pleuritic pain or rubbing heard by a physician or evidence of pleural } \\
\text { effusion } \\
\qquad \text { OR } \\
\text { Pericarditis - documented by electrocardigram or rub or evidence of pericardial effusion }\end{array}$ \\
\hline 7. Renal disorder & $\begin{array}{l}\text { Persistent proteinuria }>0.5 \text { grams per day or }>3+\text { if quantitation not performed } \\
\qquad \text { OR } \\
\text { Cellular casts - may be red cell, hemoglobin, granular, tubular, or mixed }\end{array}$ \\
\hline 8. Neurological disorder & $\begin{array}{l}\text { Seizures: in the absence of offending drugs or known metabolic derangements; e.g., uremia, } \\
\text { ketoacidosis, or electrolyte imbalance } \\
\qquad \text { OR } \\
\text { Psychosis: in the absence of offending drugs or known metabolic derangements, e.g., uremia, } \\
\text { ketoacidosis, or electrolyte imbalance }\end{array}$ \\
\hline 9. Haematolgical disorder & $\begin{array}{l}\text { Hemolyticanemia }- \text { with reticulocytosis } \\
\qquad \text { OR } \\
\text { Leukopenia }-<4,000 / \mathrm{mm}^{3} \text { on } \geq 2 \text { occasions } \\
\qquad \text { OR } \\
\text { Lyphopenia }-<1,500 / \mathrm{mm}^{3} \text { on } \geq 2 \text { occasions } \\
\qquad \text { OR } \\
\text { Thrombocytopenia }-<100,000 / \mathrm{mm}^{3} \text { in the absence of offending drugs }\end{array}$ \\
\hline 10. Immunological disorder & $\begin{array}{l}\text { Anti-DNA: antibody to native DNA in abnormal titer } \\
\text { OR } \\
\text { Anti-Sm: presence of antibody to Sm nuclear antigen } \\
\text { OR } \\
\text { Positive finding of antiphospholipid antibodies on: } \\
\text { 1. an abnormal serum level of IgG or Ig Manticardiolipin antibodies, } \\
\text { 2. a positive test result for lupus anticoagulant using a standard method, or } \\
\text { 3. a false-positive test result for at least } 6 \text { months confirmed by Treponemapallidum } \\
\text { immobilization or fluorescent treponemal antibody absorption test }\end{array}$ \\
\hline 11. Positive antinuclear antibody & $\begin{array}{l}\text { An abnormal titer of antinuclear antibody by immunofluorescence or an equivalent assay at any } \\
\text { point in time and in the absence of drugs }\end{array}$ \\
\hline
\end{tabular}


a risk during pregnancy include previous poor obstetric history (severe preeclampsia or HELLP syndrome), renal (a baseline creatinine of $>2.8$ $\mathrm{mg} / \mathrm{dL}$ ) or cardiac involvement, pulmonary hypertension, interstitial lung disease with a forced vital capacity of $<1$ litre, active disease at conception, high dose steroid therapy, and presence of aPL and nuclear antigens $(\mathrm{Ro}, \mathrm{La})^{13}$. In patients with such risk factors, especially the presence of active nephritis or pulmonary hypertension pregnancy should be avoided. In early pregnancy in the presence of these risk factors, termination of pregnancy should be discussed.

Neonatal lupus is due to passively transferred autoantibodies. It is seen in women with anti Ro or anti La antibodies with a risk of fetal heart block in $2 \%$ of such women. Which accounts for $90-95 \%$ cases of fetal or neonatal heart block. Transient neonatal cutaneous lupus is also a feature.

Management of SLE during pregnancy includes monitoring of disease activity and treatment of active disease in the event of exacerbations. Monitoring should be commenced at the beginning of the pregnancy and the initial assessment should include recording of blood pressure, assessment of renal function by GFR and a urine/ protein creatinine ratio, a full blood count, assessing for anti Ro and Anti La antibodies, lupus anticoagulant (LA), anticardiolipin (aCL) antibodies, anti-double stranded DNA (dsDNA) antibodies and a uric acid level. During pregnancy a full blood count should be performed at least monthly and GFR, and anti-dsDNA antibodies should be performed at least once every trimester, especially in women with renal involvement ${ }^{14}$. Fetal monitoring should include regular growth scans which should be tailored to the individual risk, with those with high risk factors, undergoing monthly scans from 20 weeks. If growth restriction is suspected, umbilical artery doppler should be performed. Weekly auscultation of the fetal heart should be undertaken from 16 weeks of gestation in the presence of Anti Ro of Anti-La antibodies. Treatment of fetal heart block is difficult. Trials of high dose corticosteroids, immunoglobulin and plasmapheresis have been disappointing and the disease has a high mortality ${ }^{15}$. If the fetus is viable, delivery followed by cardiac pacing should be considered.

The UK National Institute of Clinical Excellence (NICE) recommend starting low dose aspirin in women with SLE. As some women will experience exacerbations during the postpartum period, periodic assessment is recommended during the pueperium. A urinalysis and a urine protein to creatinine ratio, full blood count and anti-dsDNA antibodies are recommended during these assessments.

Treatment of active disease during pregnancy is often complex due to the harmful effects of the medication on the fetus. Although they may reduce fertility, NSAIDs are considered safe during the late first and second trimesters but should be discontinued in the third trimester. Hydroxychloroquine is considered to be safe during pregnancy. Prednisolone and methylprednisolone cross the placenta minimally while dexamethasone and betamethasone can reach the fetus at much higher concentrations. Glucocorticosteroids are relatively safe during pregnancy though an association with cleft palate has been described. The treatment aim should be to control the disease with the minimal dose of corticosteroids and at doses less than $10 \mathrm{mg}$ of prednisolone per day, no major adverse effects have been noted. Women on steroids should be periodically screened for gestational diabetes. Azathioprine may be used with caution during pregnancy but should be used in the first trimester only in severe disease. Mycophenolate and methotrexate should not be used in the pregnancy due to their teratogenic effects. Cyclophosphamide should be used only in life threatening disease of the mother and often such use may result in fetal demise either due to disease severity or the effects of the medication $^{16}$. Data on newer drugs is scarce. During the postpartum period the treatment is similar to that of a non-pregnant patient with special advise on breastfeeding depending on the drug used.

\section{Antiphospholipid syndrome}

Antiphospholipid syndrome (APS) is an autoimmune disease that increases the tendency to form blood clots and is associated with poor obstetric outcome. It demonstrates a wide spectrum of manifestations and is diagnosed in the presence of clinical criteria which includes arterial or venous thrombosis or poor obstetric history (recurrent early pregnancy loss, late pregnancy loss or placenta mediated pregnancy complications) in the presence of antiphospholipid antibodies as shown in Box $2^{17}$. APS can occur as a primary disease or secondary to autoimmune conditions such as SLE. The prevalence of primary APS in the general population is estimated to be around $0.5 \%{ }^{18}$, while APS secondary to SLE is found with a higher prevalence (up to $35 \%)^{19}$. These antibodies may be prevalent in up to $5 \%$ of the general population ${ }^{20}$ but it's significance in the absence of clinical features of APS is unknown.

Pregnancy complications associated with APS include preeclampsia, severe growth restriction, placental abruption and intrauterine fetal death ${ }^{21}$. The exact mechanism of the pathophysiology in APS is not clearly understood. Animal studies have demonstrated that in addition to thrombosis within the uteroplacental circulation ${ }^{22}$ the aPL antibodies directly bind to the trophoblastic cells and cause cellular injury, defective tissue invasion and a local inflammatory response triggered by classical and alternative complement pathways ${ }^{23}$.

Many studies and subsequent systematic reviews have demonstrated a beneficial effect of aspirin and LMWH treatment in prevention of early pregnancy loss in individuals affected by APS $^{24}$. However, the efficacy of such treatment in prevention of late pregnancy complications has not been clearly demonstrated ${ }^{25}$. New 


\section{Box 2. Revised classification criteria for the antiphospholipid syndrome ${ }^{17}$}

Antiphospholipid antibody syndrome (APS) is present if at least one of the clinical criteria and one of the laboratory criteria that follow are met

\section{Clinical criteria}

1. Vascular thrombosis

One or more clinical episodes of arterial, venous, or small vessel thrombosis

\section{Pregnancy morbidity}

(a) One or more unexplained deaths of a morphologically normal fetus at or beyond the 10th week of gestation

OR

(b) One or more premature births of a morphologically normal neonate before the 34th week of gestation because of eclampsia or severe pre-eclampsia or recognized features of placental insufficiency

OR

(c) Three or more unexplained consecutive spontaneous abortions before the 10th week of gestation

\section{Laboratory criteria}

1. Lupus anticoagulant (LA) present in plasma, on 2 or more occasions at least 12 wks apart

2. Anticardiolipin (aCL) antibody of $\lg G$ and/or IgMisotype in serum or plasma, present in medium or high titer (i.e. $>40 \mathrm{GPL}$ or MPL, or >the 99th percentile), on 2 or more occasions, at least 12 wks apart

3. Anti-beta2 glycoprotein-I antibody of IgG and/or IgMisotype in serum or plasma (in titer>the 99th percentile), present on 2 or more occasions, at least 12 wks apart

evidence is emerging that such treatment may be beneficial in prevention of placental mediated adverse events such as early onset severe preeclampsia. It is recommended that combined treatment be continued throughout the pregnancy to reduce these late pregnancy complications. Heparin should be discontinued at the onset of labour due to safety issues related to regional anaesthesia such as spinal or epidural blockade and to minimise the risk of postpartum haemorrhage. Elective induction of labour should be considered, particularly in patients on therapeutic doses of heparin. Low dose aspirin on the other hand does not carry a similar risk and maybe continued until the end of the pregnancy. Prophylactic LMWH should be recommenced in the immediate postpartum period after a lapse of at least 12 hours from insertion of spinal needle or removal of the epidural catheter in order to minimise the risk of maternal venous thromboembolism. Such treatment is continued for 6 weeks postpartum.

\section{Rheumatoid arthritis}

Rheumatoid arthritis is a complex autoimmune inflammatory condition which causes synovial inflammation in a symmetrical pattern leading to matrix destruction including damage to bone and cartilage. It is known to affect around $1 \%$ of the population and shows predominance ( 2.5 to 1 ) in females ${ }^{26}$. Therefore it is often encountered in routine obstetric practice. Due to it's high prevalence among females it has been suggested that sex hormones may have role in disease progression of rheumatoid arthritis and it has been observed that symptoms of affected women often alter between the follicle and luteal phase of the menstrual cycle ${ }^{27}$.

Pregnancy causes endocrine changes as well as modifications in the immune system. The levels of adrenal androgen levels such as dehydroepiandrosterone (DHEA), cortisol, oestrogen and progesterone levels are elevated during pregnancy. The changes in the immune system include a diminished activity of natural killer cells, an increase in soluble TNF- $\alpha$ receptors, an increase in serum plasma levels of interleukin (IL)-1 receptor antagonists and a shift of the cytokines from Th- 1 to Th-2, all of which tend to diminish disease activity in rheumatoid arthritis.

Majority of patients (66-75\%) experience symptom improvement during pregnancy ${ }^{28,29}$. The improvement in symptoms often begins in the first trimester and lasts up to a few weeks into the postpartum period. However, difficulties arise in the event of exacerbations, as similar to other connective tissue disorders, there is considerable overlap between symptoms and physiological changes in pregnancy. Disease flares are observed in a significant proportion of women in the postpartum period. The postpartum stage is also recognised as a high risk period for the initial presentation of RA and this risk is highest following the first childbirth ${ }^{30}$. Though breastfeeding is suggested as a risk factor for disease exacerbations thought to be mediated by mechanisms related to elevated prolactin levels, such hypotheses lack definitive evidence.

In contrast to other autoimmune connective tissue disorders, RA was considered to have minimal effects on pregnancy and the offspring till recent times. This has been a difficult area of study since the number of patients managed by a particular centre is often small and of those cases only a smaller proportion would develop complications in either the mother or 
the baby. However, recent evidence derived from multicentre studies as well as national birth data from many countries has shown associations between RA and pregnancy complications such as low birth weight, small for gestational age infants, premature delivery and a higher rate of caesarean section ${ }^{31-33}$. Though there has been some reports of an increased number of congenital anomalies, such as limb reduction defects and cleft palate, associated with RA, it is not very clear if such effects are due to the disease or the medication used in treatment ${ }^{31}$.

Treatment of RA during pregnancy is complicated by theteratogenic effects of the medication used. Many drugs described in the treatment of SLE are used in management of RA with similar restrictions and cautions. Additional drugs used in RA include sulfasalazine, which can be safely used in pregnancy and TNF inhibitors, in which the risk of congenital defects in not clearly understood. Therefore, TNF inhibitor use should be restricted to disease that is poorly controlled with other medication. Leflunomide, which is a disease modifying anti-rheumatic drug (DMARD), commonly used in the treatment of RA outside pregnancy has the potential for serious maternal and fetal complications. Therefore, as with methotrexate, such treatment should be stopped at least three months prior to conception and be avoided during pregnancy.

\section{Systemic sclerosis}

Systemic sclerosis (SSc) is a multisystem disease of unknown aetiology. It is characterised by overproduction of normal connective tissue with wide spread vascular damage and microvascular obliteration. The pathogenesis is believed to include inflammation, immune dysfunction, endothelial damage and cytokine and growth factor activation in the connective tissues ${ }^{34}$. The excess fibrosis and vascular damage may lead to thickening of the skin, telangiectasia, digitalischaemia with ulcers, muscle wasting, dysphagia, malabsorption, diarrhoea, cardiac arrhythmias, cardiac failure, pulmonary hypertension and renal failure. The associated changes in the skin are often referred to as scleroderma and may occur either in isolation or with involvement of other systems.

Due to its effect on multiple systems, SSc can complicate pregnancy in numerous ways. Possible complications of SSc in pregnancy include malignant hypertension due to renal involvement, rapidly worsening pulmonary hypertension, myocarditis and cardiac failure, dysphagia and oesophagitis leading to malnutrition, reduced mobility and difficulties in venous access due to skin changes, difficulties in anaesthesia, uterine and cervical changes leading to difficulties at delivery and risk of postpartum acceleration of scleroderma. The small number of patients, is a major limitation in drawing definitive conclusions regarding the effects of pregnancy on disease activity. Case reports of scleroderma renal crisis in pregnancy are reported but it is not clear if pregnancy exacerbates such events as some studies failed to demonstrate an association ${ }^{35}$.

Pregnancy was considered to pose an unacceptable risk to the woman's life and clinicians used to advice against pregnancy or recommended termination in the event a pregnancy. However, recent evidence has emerged regarding outcome of pregnancies in women with SSc, suggesting that with careful monitoring and appropriate treatment interventions, most women could go through pregnancy and delivery without major complications ${ }^{36}$. However, the risk of preterm labour, small for gestational age babies and risk of neonatal death does appear to be higher among women with SSc compared to normal controls ${ }^{37}$. As the risk of deterioration has been shown to be higher with active disease, women should be advised to delay pregnancy until adequate disease control is achieved. Scleroderma renal crisis is a life threatening complication and aggressive blood pressure control is required with ACE inhibitors. Inspite of the significant risks of ACE inhibitors to the fetus such as fetal renal dysfunction, especially in the third trimester, it may be life saving in controlling blood pressure.

Pregnancy may not be advisable in the presence of significant visceral involvement. The factors that are known to be associated with major adverse outcomes in pregnancy include severe cardiomyopathy (ejection fraction $<30 \%$ ) pulmonary hypertension, severe restrictive lung disease (forced vital capacity $<50 \%$ ) and renal insufficiency ${ }^{38}$.

The main modes of treatment in SSc are immunomodulation and antifibrotic treatment. However, as different organs are involved to varying degrees, an organ based treatment approach is often used. Modifications of the standard treatment maybe required during pregnancy due to effects on the fetus. Skin manifestations are often treated with ultraviolet light or local application of potent glucocorticosteroids. Topic steroid application is considered to be safe in pregnancy and during breastfeeding. The proton pump inhibitors used to treat oesophageal reflux disease include omeprazole, lansoprazole and pantoprazole. Their teratogenic potential has been studied in a study involving 295 pregnancies, which failed to show any association with structural defects. They are thus considered to be safe ${ }^{39}$. Antimalarial drugs (e.g. Hydroxycholoquine) as well as intravenous immunoglobulins are also considered to be safe during pregnancy. Cyclophosphamide is contraindicated in pregnancy due to its potential risk of teratogenicity (up to $20 \%$ ), especially when used in the first trimester ${ }^{40}$. Newer treatment modalities, such as low dose cyclosporine A, are emerging but their safety has yet to be fully established ${ }^{41}$.

\section{Conclusion}

Pregnancy complicated by autoimmune connective tissue disorders is not uncommon as these conditions have a high prevalence among women of reproductive age. Due to the multivisceral involvement of these conditions compounded by changes 
in disease pattern due to immune modulatory changes that accompany pregnancy, significant morbidity is expected during pregnancy. Preconceptional counselling forms the cornerstone of a successful pregnancy outcome while regular monitoring of disease activity during pregnancy will identify early exacerbations which would enable timely interventions. Obstetricians should be familiar with these conditions in order to successfully manage them during pregnancy with the help of physicians specialised in management of such conditions.

\section{REFERENCES}

1. Tan EM, Cohen AS, Fries JF, et al. The 1982 revised criteria for the classification of systemic lupus erythematosus. Arthritis Rheum 1982; 25: 1271-7.

2. Hochberg MC. Updating the American College of Rheumatology revised criteria for the classification of systemic lupus erythematosus. Arthritis Rheum 1997; 40: 1725-5. doi:10.1002/art.1780400928

3. Ulff-Møller CJ, Nielsen NM, Rostgaard $\mathrm{K}$, et al. Epstein-Barr virus-associated infectious mononucleosis and risk of systemic lupus erythematosus. Rheumatology (Oxford) 2010; 49: 1706-12. doi: 10.1093/rheumatology/keq148

4. Tsokos GC. Systemic lupus erythematosus. N Engl J Med 2011; 365: 2110-21. doi:10.1056/NEJMra1100359

5. Petri M, Howard D, Repke J. Frequency of lupus flare in pregnancy. The Hopkins Lupus Pregnancy Center experience. Arthritis Rheum 1991;34:1538-45.

6. Lockshin MD, Reinitz E, Druzin ML, et al. Lupus pregnancy. Case-control prospective study demonstrating absence of lupus exacerbation during or after pregnancy. Am J Med 1984; 77: 893-8.

7. Hayslett JP. Maternal and fetal complications in pregnant women with systemic lupus erythematosus. Am J Kidney Dis 1991; 17: $123-6$

8. Bobrie $G$, Liote $F$, Houillier $P$, et al. Pregnancy in lupus nephritis and related disorders. Am J Kidney Dis 1987; 9: 339-43.

9. Buyon JP, Cronstein BN, Morris M, et al.
Serum complement values ( $\mathrm{C} 3$ and $\mathrm{C} 4$ ) to differentiate between systemic lupus activity and pre-eclampsia. Am J Med 1986; 81: 194-200.

10. Clowse MEB. Lupus Activity in Pregnancy. Rheum Dis Clin North Am. 2007; 33 : 237-52.doi:10.1016/j.rdc. 2007. 01.002

11. Yasmeen S, Wilkins EE, Field NT, et al. Pregnancy outcomes in women with systemic lupus erythematosus. Journal of Maternal-Fetal Medicine. 2001; 10: 91-6.

12. Opatrny L, David M, Kahn SR, et al. Association between antiphospholipid antibodies and recurrent fetal loss in women without autoimmune disease: a metaanalysis. J Rheumatol 2006; 33: 2214-21.

13. Ruiz-Irastorza G, Lima F, Alves J, et al. Increased rate of lupus flare during pregnancy and the puerperium: a prospective study of 78 pregnancies. $\mathrm{Br} \mathrm{J}$ Rheumatol 1996; 35: 133-8.

14. Lockshin MD, Sammaritano LR. Lupus pregnancy. Autoimmunity 2003; 36: 33-40.

15. Capone C, Buyon JP, Friedman DM, et al. Cardiac Manifestations of Neonatal Lupus: A Review of Autoantibody-associated Congenital Heart Block and its Impact in an Adult Population. Cardiol Rev 2012; 20: 72 6. doi:10.1097/CRD.0b013e31823c808b

16. Clowse MEB, Magder L, Petri M. Cyclophosphamide for lupus during pregnancy. Lupus 2005; 14: 593-7.

17. Miyakis S, Lockshin MD, Atsumi T, et al. International consensus statement on an update of the classification criteria for definite antiphospholipid syndrome (APS). 2006. 295-306. doi:10.1111/j.1538-7836. 2006. 01753.x.

18. Cohen D, Berger SP, Steup-Beekman $\mathrm{GM}$, et al. Diagnosis and management of the antiphospholipid syndrome. BMJ 2010; 340: c2541.

19. Levine JS, Branch DW, Rauch J. The Antiphospholipid Syndrome. N Engl J Med 2002; 346: 752-63. doi:10.1056/NEJ Mra 002974.

20. Danza A, Ruiz-Irastorza G, Khamashta M. Antiphospohlipid syndrome in obstetrics. Best Pract Res Clin Obstet Gynaecol 2012;26:65-76. doi:10.1016/j. bpobgyn. 2011.10.006.

21. Rodger MA. An update on thrombophilia and placenta mediated pregnancy complications: what should we tell our patients? Thromb Res 2013; 131 Suppl 1: S25-7.doi:10.1016/S0049-3848(13)70015-X.

22. Branch DW. Antiphospholipid antibodies and fetal compromise. Thromb Res 2004;114: 415-8. doi:10.1016/j. thromres. 2004.08.005.

23. Greer IA. Thrombophilia: implications for pregnancy outcome. Thromb Res 2003; 109: 73-81. doi:10.1016/S0049-3848 (03) 00095-1

24. Empson $\mathrm{M}$, Lassere $\mathrm{M}$, Craig $\mathrm{J}$, et al. Prevention of recurrent miscarriage for women with antiphospholipid antibody or lupus anticoagulant. Cochrane Database Syst Rev 2005; :CD002859. doi:10.1002/ 14651858.CD002859.pub2

25. Branch DW, Silver RM, Porter TF. Obstetric antiphospholipid syndrome: current uncertainties should guide our way. Lupus 2010;19:446-52. doi:10.1177/ 0961203310361490

26. Dugowson CE, Koepsell TD, Voigt LF, et al. Rheumatoid arthritis in women. Incidence rates in group health cooperative, Seattle, Washington, 1987-1989. Arthritis Rheum 1991; 34: 1502-7.

27. Case AM, Reid RL. Effects of the menstrual cycle on medical disorders. Arch Intern Med 1998; 158: 1405-12.

28. Persellin $\mathrm{RH}$. The effect of pregnancy on rheumatoid arthritis. Bull Rheum Dis 1976; 27: 922-7.

29. Ostensen M. The influence of pregnancy on blood parameters in patients with rheumatic disease. Scand J Rheumatol 1984; 13: 203-8.

30. Silman A, Kay A, Brennan P. Timing of pregnancy in relation to the onset of rheumatoid arthritis. Arthritis Rheum 1992; 35: 152-5.

31. Chakravarty EF. Rheumatoid arthritis and pregnancy: where do we go from here? J Intern Med 2010;268:309-11. doi:10.1111/ j. 1365-2796.2010.02250.x

32. Chakravarty EF. Rheumatoid arthritis and pregnancy: beyond smaller and preterm babies. Arthritis Rheum 2011; 63: 1469-71. doi:10.1002/art.30206

33. Lin $\mathrm{H}-\mathrm{C}$, Chen $\mathrm{S}-\mathrm{F}$, Lin $\mathrm{H}-\mathrm{C}$, et al. Increased risk of adverse pregnancy outcomes in women with rheumatoid arthritis: a nationwide population-based study. Ann Rheum Dis 2010;69:715-7. doi:10.1136/ ard.2008.105262 
34. Black CM. Systemic sclerosis and pregnancy. Baillieres Clin Rheumatol 1990; 4: 105-24.

35. Steen VD, Conte C, Day N, et al. Pregnancy in women with systemic sclerosis. Arthritis Rheum 1989; 32: 151-7.

36. Chakravarty EF. Vascular Complications of Systemic Sclerosis during Pregnancy. Int J Rheumatol 2010; 2010. doi:10.1155/2010/287248

37. Steen VD, Medsger TA. Fertility and pregnancy outcome in women with systemic sclerosis. Arthritis Rheum 1999; 42: 763-8. doi:10.1002/1529-0131 (199904) 42: 4<763: AID-ANR21 >3.0.CO;2-V

38. Steen VD. Pregnancy in Scleroderma. Rheum Dis Clin North Am. 2007; 33: 345-58. doi:10.1016/j.rdc.2007.03.001

39. Diav-Citrin O, Arnon J, Shechtman S, et al. The safety of proton pump inhibitors in pregnancy: a multicentre prospective controlled study. Aliment Pharmacol Ther
2005; 21: 269-75. doi:10.1111/j.1365-2036. 2005.02306.x

40. Ostensen M, Khamashta M, Lockshin M, et al. Anti-inflammatory and immunosuppressive drugs and reproduction. Arthritis Res Ther 2006; 8: 209. doi:10.1186/ar1957.

41. Basso M, Ghio M, Filaci G, et al. A case of successful pregnancy in a woman with systemic sclerosis treated with cyclosporin. Rheumatology (Oxford) 2004; 43: 1310-1. doi:10.1093/rheumatology/keh319. 